\title{
Discriminative validity of the lower and upper quarter $Y$ balance test performance: a comparison between healthy trained and untrained youth
}

\author{
Gerrit Schwiertz $^{1 *}$, Rainer Beurskens ${ }^{2+}$ and Thomas Muehlbauer ${ }^{1+}$
}

\begin{abstract}
Background: The Lower (YBT-LQ) and Upper (YBT-UQ) Quarter Y Balance Test have been widely used for the assessment of dynamic balance and shoulder mobility/stability, respectively. However, investigations on the validity of the two tests in youth are lacking. Therefore, we performed two studies to determine discriminative validity of the YBT-LQ (study 1) and the YBT-UQ (study 2) in healthy youth.

Methods: Sixty-nine male soccer players (age: $14.4 \pm 1.9 \mathrm{yrs})$ and 69 age-matched untrained male subjects (14.3 \pm $1.6 \mathrm{yrs}$ ) participated in study 1 and 37 young swimmers (age: $12.3 \pm 2.1 \mathrm{yrs)}$ ) as well as 37 age-/sex-matched individuals (age: $12.5 \pm 2.0 \mathrm{yrs}$ ) took part in study 2. Absolute $(\mathrm{cm})$ and relative (\% leg/arm length) maximal reach distances per reach direction and the composite score of the YBT-LQ/UQ were used as outcome measures. Oneway analysis of variance and the receiver operator characteristic curve analysis (i.e., calculating the area under the curve $[A \cup C]$ ) were conducted to assess discriminative validity.

Results: Concerning the relative values, youth athletes showed significantly better YBT-LQ (study 1: $p<0.001, d=$ 0.86-1.21) and YBT-UQ (study 2: $p<0.001, d=0.88-1.48$ ) test performances compared to age- and sex-matched untrained subjects. Further, AUC-values indicated a chance of $\geq 74 \%$ (YBT-LQ) and $\geq 71 \%$ (YBT-UQ) to discriminate between youth athletes and controls. These findings were confirmed when using the absolute data for analysis.

Conclusions: According to our results, the YBT-LQ and the YBT-UQ seem to be useful test instruments to discriminate trained and untrained healthy youth performance for dynamic balance and shoulder mobility/stability, respectively.
\end{abstract}

Keywords: Postural control, Shoulder mobility/stability, Young athletes, Validation

\footnotetext{
* Correspondence: gerrit.schwiertz@uni-due.de

${ }^{\dagger}$ Rainer Beurskens and Thomas Muehlbauer are co-authors.

${ }^{1}$ Division of Movement and Training Sciences/Biomechanics of Sport, University of Duisburg-Essen, Gladbecker Str. 182, 45141 Essen, Germany

Full list of author information is available at the end of the article
}

(c) The Author(s). 2020 Open Access This article is licensed under a Creative Commons Attribution 4.0 International License, which permits use, sharing, adaptation, distribution and reproduction in any medium or format, as long as you give appropriate credit to the original author(s) and the source, provide a link to the Creative Commons licence, and indicate if changes were made. The images or other third party material in this article are included in the article's Creative Commons licence, unless indicated otherwise in a credit line to the material. If material is not included in the article's Creative Commons licence and your intended use is not permitted by statutory regulation or exceeds the permitted use, you will need to obtain permission directly from the copyright holder. To view a copy of this licence, visit http://creativecommons.org/licenses/by/4.0/. The Creative Commons Public Domain Dedication waiver (http://creativecommons.org/publicdomain/zero/1.0/) applies to the data made available in this article, unless otherwise stated in a credit line to the data. 


\section{Background}

The Y-Balance-Test (YBT) represents a field-based test $[1,2]$ assessing i) dynamic balance performance when applied to lower extremities (YBT-LQ) [3] and ii) shoulder mobility/stability when applied to upper extremities (YBT-UQ) [4]. Both testing procedures have widely been used in previous literature to determine influencing factors such as age $[5,6]$, sex $[6,7]$, anthropometric characteristics $[8,9]$, limb dominance/strength $[4,10]$, and previous injuries $[11,12]$ and to detect performance differences in different cohorts [3, 13-15].

Concerning the latter aspect, Butler et al. [3] investigated YBT-LQ performance in male high school, collegiate, and professional soccer players and detected significantly lower reach distances in the posteromedial and posterolateral directions for the high school players than the two other groups of player. Further, Bullock et al. [13] studied middle school, high school, college, and professional basketball players and observed that high school players performed significantly better (i.e., anterior reach direction) compared to middle school and college players. Regarding the YBT-UQ, Bullock et al. [14] tested high school and collegiate swimmers and found significantly better values for the medial reach direction in favour of the latter group of swimmers. In another study, Krysak et al. [15] compared middle school, high school, college, and professional golfer players and reported greater reach distances in the medial, inferolateral, and superolateral directions for the professional golfers compared to the three other groups.

Results of the aforementioned studies shed light onto performance differences based on the athletes' level of competition and experience. However, not only their level of competition but also the age differed between cohorts. For example, in the study conducted by Butler and colleagues [3], age differed between 15.6 years in high school soccer players and 26.2 years in professional soccer players. Further, participants in Krysak and coworkers [15] were aged between 12.2 years (middle school golfers) und 31.8 years (professional golfers). Thus, participants' age might have influenced performance differences in these studies and it remains unclear whether the YBT-LQ/UQ is discriminatively valid. As a consequence, studies comparing age- and sex-matched persons with various levels of competition are needed.

Most notably, examining discriminative validity of the YBT-LQ/UQ in children and adolescents is important since these age groups are used for talent selection and scouting [16]. More precisely, the investigation of age -/sex-matched youth in relation to their training status is useful to discriminate high-performer versus lowperformer using the YBT-LQ/UQ. Thus, the aim of the present study was to determine discriminative validity of the YBT-LQ (study 1) and the YBT-UQ (study 2) by comparing age- and sex-matched trained versus untrained youth. With reference to the relevant literature [3, 13-15], we expected better performances in both tests for trained compared to untrained youth and we hypothesized good discriminative validity for both tests.

\section{Methods \\ Participants}

Participants' characteristics are summarized in Table 1. In study 1, 69 male soccer players from a local sports club and 69 age-matched untrained male subjects performed the YBT-LQ. In study 2, 37 female and male swimmers from a local sports club and 37 age-/sexmatched untrained individuals conducted the YBT-UQ. The maturity offset was calculated in terms of years from peak height velocity (PHV) for each participant by using the formula provided by Moore et al. [17]. Participants' assent and parents' written informed consent were obtained prior to the start of the study. The Human Ethics Committee at the University of Duisburg-Essen, Faculty of Educational Sciences approved the study protocol.

\section{Testing procedures}

Discriminative validity of the YBT-LQ and the YBT-UQ was assessed in study 1 and study 2, respectively. In both studies, we used a standardized general warm-up comprising 5 min of running at a moderate speed and a test-specific warm-up consisting of three submaximal reaches per arm/leg and reach direction. All participants received standardized verbal instructions and a visual demonstration regarding the testing procedure that included assessment of anthropometric variables (i.e., body mass, body height, arm length $[\mathrm{AL}]$, leg length [LL]) followed by performance assessment in the YBT-LQ (study 1) or YBT-UQ (study 2). The participants had no prior experience with the YBT-LQ/UQ.

\section{Assessment of anthropometric variables}

Body mass $(\mathrm{kg})$ was measured in light clothing and without shoes to the nearest $100 \mathrm{~g}$ with an electronic scale (seca 803, Basel, Switzerland). Further, body height $(\mathrm{cm})$ was determined without shoes to the nearest $0.5 \mathrm{~cm}$ with a stadiometer (seca 217, Basel, Switzerland). Body mass index was calculated using body mass divided by height squared $\left(\mathrm{kg} / \mathrm{m}^{2}\right)$. Length $(\mathrm{cm})$ of the right and left arm was determined with a cloth tape measure from the seventh cervical spinous process to the distal tip of the middle finger with the shoulder being in a $90^{\circ}$ abduction [18]. Further, left and right leg length $(\mathrm{cm})$ were assessed by measuring the distance from the anterior superior iliac spine to the most distal aspect of the medial malleolus using a cloth tape with the participant lying supine [19]. In accordance to Fusco et al. [8], trunk length was 
Table 1 Characteristics of the participants by study

\begin{tabular}{|c|c|c|c|c|c|c|}
\hline \multirow[t]{2}{*}{ Characteristic } & \multicolumn{3}{|l|}{ Study $1(N=138)$} & \multicolumn{3}{|l|}{ Study $2(N=74)$} \\
\hline & Soccer players $(n=69)$ & Controls $(n=69)$ & $p$-value & Swimmers $(n=37)$ & Controls $(n=37)$ & $p$-value \\
\hline Age (yrs) & $14.4 \pm 1.9$ & $14.3 \pm 1.6$ & .511 & $12.3 \pm 2.1$ & $12.5 \pm 2.0$ & .650 \\
\hline $\operatorname{Sex}(f / m)$ & $0 / 69$ & $0 / 69$ & & $22 / 15$ & $22 / 15$ & \\
\hline Body mass (kg) & $60.9 \pm 14.8$ & $64.8 \pm 15.8$ & .136 & $49.2 \pm 14.8$ & $52.6 \pm 17.8$ & .378 \\
\hline Body height $(\mathrm{cm})$ & $169.6 \pm 12.5$ & $173.5 \pm 12.4$ & .069 & $160.8 \pm 14.7$ & $160.5 \pm 14.7$ & .928 \\
\hline $\mathrm{BMI}\left(\mathrm{kg} / \mathrm{m}^{2}\right)$ & $20.8 \pm 2.9$ & $21.3 \pm 4.0$ & .419 & $18.6 \pm 2.5$ & $19.8 \pm 3.3$ & .079 \\
\hline Maturity offset (yrs from PHV) & $1.0 \pm 1.5$ & $0.9 \pm 2.7$ & .750 & $0.1 \pm 2.0$ & $0.3 \pm 1.9$ & .618 \\
\hline Left leg length (cm) & $90.2 \pm 6.2$ & $94.1 \pm 7.7$ & .196 & - & - & - \\
\hline Right leg length (cm) & $90.1 \pm 6.1$ & $94.0 \pm 7.5$ & .184 & - & - & - \\
\hline Left arm length (cm) & - & - & - & $81.8 \pm 8.7$ & $81.0 \pm 8.2$ & .686 \\
\hline Right arm length $(\mathrm{cm})$ & - & - & - & $81.9 \pm 8.8$ & $81.2 \pm 8.3$ & .706 \\
\hline Trunk length (cm) & $79.8 \pm 8.3$ & $80.2 \pm 8.2$ & .419 & - & - & - \\
\hline Relative lower limb length (\%) & $53.1 \pm 2.0$ & $54.0 \pm 2.8$ & .504 & - & - & - \\
\hline
\end{tabular}

Data are mean \pm standard deviation

$B M I$ body mass index, $f$ female, $m$ male, $P H V$ peak height velocity

calculated as the difference between body height and LL and relative lower limb length was determined using LL divided by body height and then multiplied by 100 .

\section{Assessment of lower quarter $Y$ balance test performance}

YBT-LQ performance was assessed by means of the YBT Kit (Functional Movement Systems ${ }^{\circ}$, Chatham, USA). The test kit consists of a centralized platform to which three pipes were attached representing the anterior (AT), posteromedial (PM), and posterolateral (PL) reach directions (Fig. 1a). Each pipe is marked in $1.0-\mathrm{cm}$ increments for measurement purposes and equipped with a moveable reach indicator. The participants were asked to move the reach indicator as far as possible into the AT direction with the right leg while standing on the centralized platform with their left leg followed by standing on the right leg and reaching with the left leg. This protocol was then replicated for the PM and PL directions. Each participant performed three practice trials followed by three datacollection trials per leg and reach direction. A oneminute rest was provided between trials. The absolute maximal reach distance $(\mathrm{cm})$ per leg and reach direction was used for further analysis. Reliability of the YBT-LQ has been shown to be predominately "excellent" in healthy youth [20].

\section{Assessment of upper quarter $Y$ balance test performance}

The YBT Kit was also used for the assessment of YBTUQ performance, with the three pipes representing the medial (MD), inferolateral (IL), and superolateral (SL) reach directions (Fig. 1b). Participants were instructed to move the reach indicator with the right arm as far as possible in the MD, IL, and SL directions while maintaining a weight bearing one-arm push-up position with their left arm on the centralized platform. This protocol was then replicated for the left arm. Three practice trials were conducted followed by three data-collection trials. The rest between trials comprised $1 \mathrm{~min}$. The best values (i.e., absolute maximal reach distance in $\mathrm{cm}$ ) per arm and reach direction was used for further analysis. The reliability of the YBT-UQ ranged from "moderateto-good" to "excellent" in healthy youth [21].

\section{Data and statistical analyses}

For both tests, relative/normalized maximal reach distances (\%) per reach direction and leg/arm were calculated by dividing the absolute maximal reach distance $(\mathrm{cm})$ by LL or AL $(\mathrm{cm})$ and then multiplying by 100 . In addition, the normalized (\%) composite score (CS) per leg/arm was computed as the sum of the absolute maximal reach distance $(\mathrm{cm})$ per reach direction divided by three times LL or AL and then multiplied by 100 .

Further, the mean value was calculated as a measure of central tendency and the standard deviation (SD) as a dispersion measure. Normal distribution was examined using the Shapiro-Wilk test $(p>0.05)$ and homogeneity of variances using the Levene test $(p>0.05)$. An independent samples t-test was used to quantify differences between the cohorts. Discriminative validity was analyzed using the one-way analysis of variance (ANOVA). Statistically significant differences were identified at $p<$ 0.05. Furthermore, effect size (Cohen's $d$ ) was calculated and classified as "small" $(0 \leq d \leq 0.49)$, "moderate" $(0.50 \leq d \leq 0.79)$, and "large" $(d \geq 0.80)$ [22]. Moreover, 


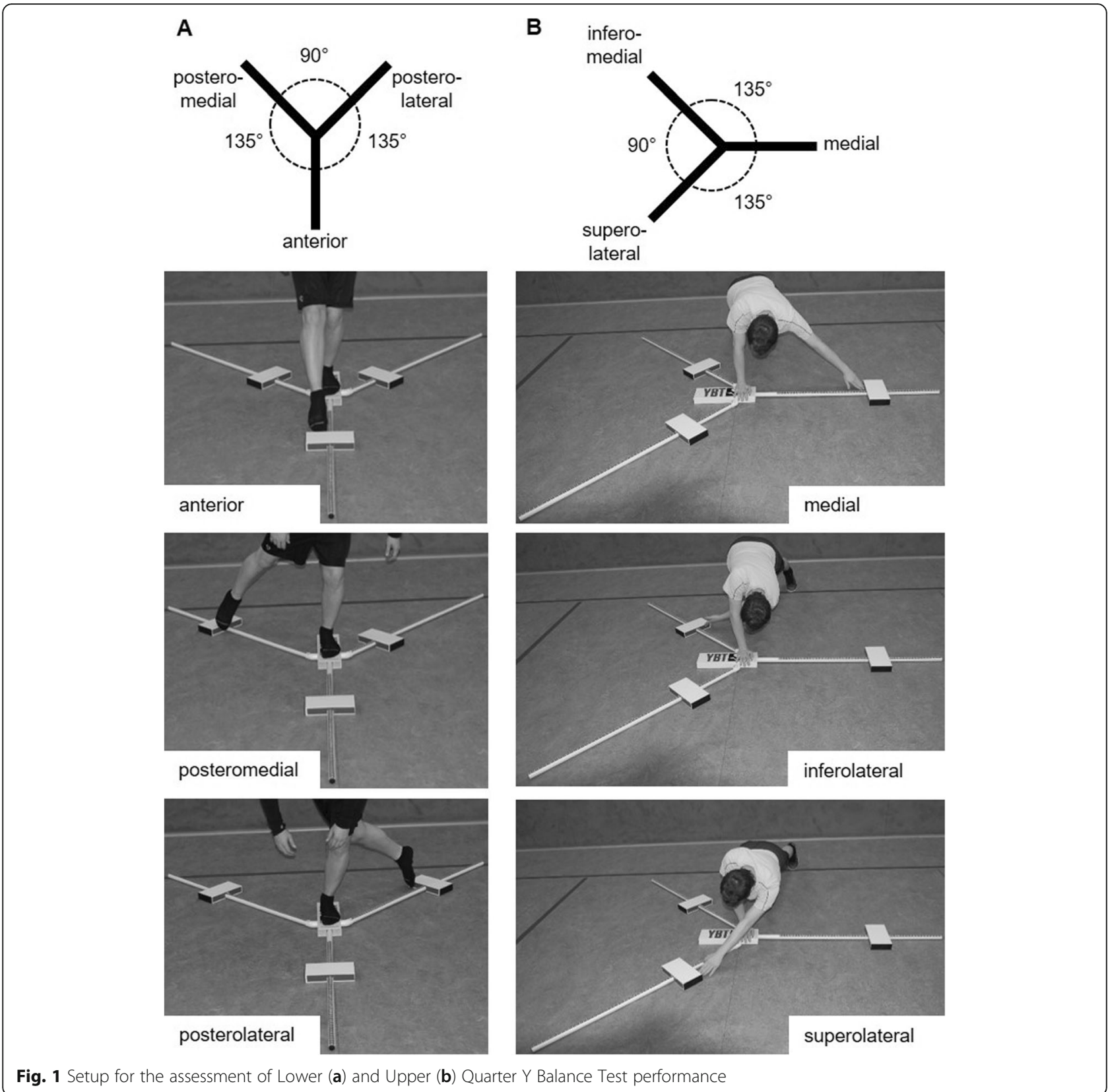

we conducted a receiver operator characteristic (ROC) curve analysis and calculated the area under the receiver operator characteristic (AUC) curve for each outcome measure (i.e., per reach direction and composite score) separately. The AUC measures the entire two-dimensional area underneath the entire ROC curve. In this regard, Deyo and Centor [23] stated that an AUC-value of 0.50 indicates "no "and an AUC-value of 1.0 indicates "perfect" discriminative validity. All statistical analyses were performed using Statistical Package for Social Sciences version 24.0 (SPSS Inc., Chicago, IL, USA).

\section{Results}

Characteristics of the study participants

Irrespective of outcome, we did not detect statistically significant differences in participants' characteristics, neither in study 1 nor in study 2 (Table 1 ).

\section{Discriminative validity of lower quarter $Y$ balance test performance (study 1 )}

Statistical data on the discriminative validity for YBTLQ performance between young male soccer players and age-matched male untrained subjects are displayed in Table 2. With regard to the absolute values (i.e., reach 
Table 2 Discriminative validity for the assessment of absolute ( $\mathrm{cm}$ ) and relative (\% leg length) Lower Quarter Y Balance Test performance between trained (i.e., soccer players) and age-matched untrained (i.e., controls) youth

\begin{tabular}{|c|c|c|c|c|}
\hline & \multicolumn{2}{|l|}{ Participants $(N=138)$} & \multicolumn{2}{|l|}{ Statistics } \\
\hline & Soccer players $(n=69)$ & Controls $(n=69)$ & $p$-value $(d)$ & AUC-value \\
\hline \multicolumn{5}{|l|}{ Right leg reach } \\
\hline AT $(\mathrm{cm})$ & $70.9 \pm 7.6$ & $67.2 \pm 7.4$ & $.004(0.49)$ & .63 \\
\hline $\mathrm{PM}(\mathrm{cm})$ & $107.6 \pm 8.5$ & $101.1 \pm 11.4$ & $<.001(0.64)$ & .67 \\
\hline $\mathrm{PL}(\mathrm{cm})$ & $103.9 \pm 7.8$ & $97.7 \pm 10.2$ & $<.001(0.69)$ & .69 \\
\hline \multicolumn{5}{|l|}{ Left leg reach } \\
\hline AT $(\mathrm{cm})$ & $69.6 \pm 7.5$ & $66.0 \pm 8.2$ & $.009(0.45)$ & .64 \\
\hline $\mathrm{PM}(\mathrm{cm})$ & $106.5 \pm 8.2$ & $99.5 \pm 13.1$ & $<.001(0.64)$ & .66 \\
\hline $\mathrm{PL}(\mathrm{cm})$ & $103.7 \pm 9.1$ & $96.8 \pm 12.2$ & $<.001(0.65)$ & .68 \\
\hline \multicolumn{5}{|l|}{ Right leg reach } \\
\hline AT (\% LL) & $78.9 \pm 9.5$ & $71.5 \pm 7.3$ & $<.001(0.87)$ & .74 \\
\hline PM (\% LL) & $119.5 \pm 8.8$ & $107.8 \pm 12.1$ & $<.001(1.11)$ & .78 \\
\hline PL (\% LL) & $115.6 \pm 9.1$ & $104.2 \pm 11.5$ & $<.001(1.10)$ & .78 \\
\hline CS (\% LL) & $104.6 \pm 8.1$ & $94.5 \pm 9.4$ & $<.001(1.16)$ & .80 \\
\hline \multicolumn{5}{|l|}{ Left leg reach } \\
\hline AT (\% LL) & $77.5 \pm 9.2$ & $70.3 \pm 7.3$ & $<.001(0.86)$ & .74 \\
\hline PM (\% LL) & $118.5 \pm 8.7$ & $106.0 \pm 12.3$ & $<.001(1.17)$ & .79 \\
\hline PL (\% LL) & $115.5 \pm 10.6$ & $103.2 \pm 12.3$ & $<.001(1.08)$ & .77 \\
\hline CS (\% LL) & $103.8 \pm 8.1$ & $93.2 \pm 9.6$ & $<.001(1.21)$ & .81 \\
\hline
\end{tabular}

Data are mean \pm standard deviation. Absolute values $(\mathrm{cm})$ are shown first followed by relative values (\% LL). Cohen's $d$ [18] can be classified as being small $(0 \leq$ $d \leq 0.49)$, medium $(0.50 \leq d \leq 0.79)$, or large $(d \geq 0.80)$. In accordance with Deyo and Centor [23], the AUC-value can lie between 0.5 ("no" discriminative validity) and 1.0 ("perfect" discriminative validity)

$A T$ anterior, AUC area under the receiver operator characteristic (ROC) curve, CS composite score, $L L$ leg length, $P L$ posterolateral, $P M$ posteromedial

distance in $\mathrm{cm}$ ), the soccer players compared to the controls achieved small- to medium-sized and significantly better values for AT $(p \leq 0.009 ; d=0.45-0.49)$, PM $(p<$ $0.001 ; d=0.64)$, and PL $(p<0.001 ; d=0.65-0.69)$ directions, irrespective of the reaching leg. In addition, we detected significant differences between the three reach directions $(\mathrm{PM}>\mathrm{PL}>\mathrm{AT})$ for the right and left leg reach, irrespective of training status (i.e., soccer players or untrained subjects). Further, AUC-values were 0.63 (AT), 0.67 (PM), and 0.69 (PL) for the right and 0.64 (AT), 0.66 (PM), and 0.68 (PL) for the left leg reach, respectively. Results indicate that there is a chance of 63$69 \%$ that the YBT-LQ is possible to differentiate between soccer players and age-matched untrained individuals.

Concerning the relative values (i.e., reach distance in \% LL), the soccer players achieved large-sized and significantly better values for AT $(p<0.001 ; d=0.86-0.87)$, PM $(p<0.001 ; d=1.11-1.17)$, and PL $(p<0.001 ; d=$ $1.08-1.10)$ directions as well as for the CS $(p<0.001$; $d=1.16-1.21)$ compared to the controls, irrespective of the reaching leg. Additionally, we found significant differences between the three reach directions $(\mathrm{PM}>\mathrm{PL}>$ AT) for the right and the left leg reach, irrespective of training status (i.e., soccer players or untrained subjects). Further, AUC-values were 0.74 (AT), 0.78 (PM), 0.78
(PL), and $0.80(\mathrm{CS})$ for the right and 0.74 (AT), 0.79 (PM), 0.77 (PL), and $0.81(\mathrm{CS})$ for the left leg reach, respectively. Results indicate that there is a chance of 74$81 \%$ that the YBT-LQ is possible to differentiate between soccer players and age-matched untrained individuals.

\section{Discriminative validity of upper quarter $Y$ balance test performance (study 2)}

Table 3 shows the statistics on the discriminative validity for YBT-UQ performance between young female and male swimmers and age-/sex-matched untrained subjects. With regard to the absolute values (i.e., reach distance in $\mathrm{cm}$ ), we detected significant, large-sized differences in favour of swimmers for the MD $(p<0.001$, $d=0.87-0.91)$, IL $(p<0.001, d=1.19-1.30)$, and SL $(p<$ $0.001, d=1.46-1.78)$ directions. Further, we found significant differences between the three reach directions (MD $>$ IL $>$ SL) for the right and left arm reach, irrespective of training status (i.e., swimmers or untrained subjects). In addition, AUC-values were 0.75 (MD), 0.83 (IL), and 0.90 (SL) for the right and 0.75 (MD), 0.79 (IL), and $0.84(\mathrm{SL})$ for the left arm reach, respectively. In other words, there is a chance of $75-90 \%$ that the YBTUQ is possible to distinguish between swimmers and age -/sex-matched untrained subjects. 
Table 3 Discriminative validity for the assessment of absolute $(\mathrm{cm})$ and relative (\% arm length) Upper Quarter Y Balance Test performance between trained (i.e., swimmers) and age-/sex-matched untrained (i.e., controls) youth

\begin{tabular}{|c|c|c|c|c|}
\hline & \multicolumn{2}{|l|}{ Participants $(N=74)$} & \multicolumn{2}{|l|}{ Statistics } \\
\hline & Swimmers $(n=37)$ & Controls $(n=37)$ & $p$-value $(d)$ & AUC-value \\
\hline \multicolumn{5}{|l|}{ Right arm reach } \\
\hline $\mathrm{MD}(\mathrm{cm})$ & $84.8 \pm 8.8$ & $76.3 \pm 10.6$ & $<.001(0.87)$ & .75 \\
\hline IL (cm) & $83.1 \pm 10.7$ & $69.7 \pm 9.8$ & $<.001(1.30)$ & .83 \\
\hline $\mathrm{SL}(\mathrm{cm})$ & $67.9 \pm 8.3$ & $53.6 \pm 7.8$ & $<.001(1.78)$ & .90 \\
\hline \multicolumn{5}{|l|}{ Left arm reach } \\
\hline $\mathrm{MD}(\mathrm{cm})$ & $83.6 \pm 8.9$ & $75.1 \pm 10.0$ & $<.001(0.91)$ & .75 \\
\hline $\mathbb{I L}(\mathrm{cm})$ & $82.8 \pm 9.7$ & $70.7 \pm 10.5$ & $<.001(1.19)$ & .79 \\
\hline $\mathrm{SL}(\mathrm{cm})$ & $64.3 \pm 7.3$ & $52.6 \pm 8.6$ & $<.001(1.46)$ & .84 \\
\hline \multicolumn{5}{|c|}{ Right arm reach } \\
\hline $\mathrm{MD}(\% \mathrm{AL})$ & $104.0 \pm 8.4$ & $94.5 \pm 11.9$ & $<.001(0.92)$ & .73 \\
\hline IL (\% AL) & $102.2 \pm 13.5$ & $87.2 \pm 16.6$ & $<.001(0.99)$ & .76 \\
\hline $\mathrm{SL}(\% \mathrm{AL})$ & $83.3 \pm 9.3$ & $67.0 \pm 12.6$ & $<.001(1.48)$ & .85 \\
\hline $\mathrm{CS}(\% \mathrm{AL})$ & $96.0 \pm 8.1$ & $82.9 \pm 12.1$ & $<.001(1.28)$ & .80 \\
\hline \multicolumn{5}{|l|}{ Left arm reach } \\
\hline $\mathrm{MD}(\% \mathrm{AL})$ & $102.3 \pm 6.8$ & $92.7 \pm 10.4$ & $<.001(1.09)$ & .78 \\
\hline IL (\% AL) & $101.8 \pm 12.9$ & $88.3 \pm 17.5$ & $<.001(0.88)$ & .71 \\
\hline $\mathrm{SL}(\% \mathrm{AL})$ & $79.0 \pm 9.4$ & $65.8 \pm 13.8$ & $<.001(1.11)$ & .77 \\
\hline $\mathrm{CS}(\% \mathrm{AL})$ & $94.8 \pm 9.0$ & $82.3 \pm 12.3$ & $<.001(1.17)$ & .79 \\
\hline
\end{tabular}

Data are mean \pm standard deviation. Absolute values $(\mathrm{cm})$ are shown first followed by relative values (\% AL). Cohen's $d$ [18] can be classified as being small ( $0 \leq$ $d \leq 0.49)$, medium $(0.50 \leq d \leq 0.79)$, or large $(d \geq 0.80)$. In accordance with Deyo and Conter [23], the AUC-value can lie between 0.5 ("no" discriminative validity) and 1.0 ("perfect" discriminative validity)

$A L$ arm length, $A U C$ area under the receiver operator characteristic (ROC) curve, CS composite score, IL inferolateral, $M D$ medial, $S L$ superolateral

With respect to the relative values (i.e., reach distance in \% AL), we observed significant, large-sized differences in favour of swimmers for the MD $(p<0.001, d=0.92-$ 1.09), IL $(p<0.001, d=0.88-0.99)$, and SL $(p<0.001$, $d=1.11-1.48)$ directions as well as for the CS $(p<0.001$, $d=1.17-1.28)$. In addition, AUC-values were $0.73(\mathrm{MD})$, 0.76 (IL), 0.85 (SL), and 0.80 (CS) for the right and 0.78 (MD), 0.71 (IL), 0.77 (SL), and 0.79 (CS) for the left arm reach, respectively. In other words, there is a chance of $71-85 \%$ that the YBT-UQ is possible to distinguish between swimmers and age-/sex-matched untrained subjects.

\section{Discussion}

To our knowledge, the present studies investigated discriminative validity of YBT-LQ/UQ performance between healthy trained and untrained youth for the first time. Main results for the relative/normalized measures (i.e., reach distance in \% LL/AL) can be summarized as follows and were confirmed by the same analyses using the absolute/raw measures (i.e., reach distance in $\mathrm{cm}$ ): (1) trained youth (i.e., soccer players and swimmers) showed large-sized significantly better YBT-LQ/UQ performance compared with age-/sex-matched untrained controls; (2) ROC analyses revealed a chance of $\geq 74 \%$ (YBT-LQ) and $\geq 71 \%$ (YBT-UQ) to discriminate youth athletes from untrained youth.

In line with our hypothesis stating better performances in both tests for trained compared to untrained youth, one-way ANOVA revealed significantly larger absolute $(\mathrm{cm})$ and relative (\% LL/AL) YBT-LQ/UQ reach distances in trained participants (i.e., soccer players and swimmers) compared to age-/sex-matched controls. This result corresponds with findings from studies [3, $13-15,24,25]$ that investigated groups of athletes with varying levels of competition (e.g., high school vs. collegiate vs. professional players) and reported better relative YBT-LQ/UQ performance for those with a higher than for those with a lower competition level. However, besides the differences in competition level, the included persons also differed in age (i.e., adults versus adolescents), which might have influenced the results. In the present study, we included age-/sex-matched controls and results nonetheless showed better absolute and relative YBT-LQ/UQ-performance in trained compared to untrained youth. This finding is in line with a study by Engquist et al. [26] that investigated young adults (mean age: $20 \pm 1.6$ years) and found larger YBT-LQ reach 
distances in trained (i.e., female Division I studentathletes) compared to non-trained but same-aged (i.e., general female college students) individuals. Our findings and the results by Engquist and colleagues indicate that differences in absolute and relative YBT-LQ/UQ performance in age- and sex-matched individuals are based on training status and competition level. Additionally and also in accordance with our hypothesis stating good discriminative validity for the YBT-LQ/UQ in healthy youth, our ROC analysis for the relative data yielded a chance between 74 to $81 \%$ (YBT-LQ) and 71 to $85 \%$ (YBT-UQ) to discriminate youth athletes from age-/sex-matched untrained youth and this was confirmed using the absolute data. Thus, YBT-LQ (i.e., dynamic balance) and YBT-UQ (i.e., shoulder mobility/ stability) demands seem to be associated with respective athletic requirements in soccer and swimming.

What might be the reason for performance differences in YBT-LQ/UQ between trained and age-/sex-matched untrained youth? One might argue that trained compared to non-trained youth possess a higher amount of long-lasting, continuous and intense training experience [27]. Another reason might be the genetic profile of trained individuals $[28,29]$. For example, Murtagh et al. [28] investigated the relationship of multiple single nucleotide polymorphisms with physical performance measures in elite male youth soccer players and control participants. The authors observed differences in the genetic profile (e.g., higher genotype frequency distribution in soccer players) and showed that physical performance was associated with some measures of the genetic profile. Taken together, both preconditions cause specific adaptations $[30,31]$ that allow for higher performances in sport-specific as well as in physical fitness measures.

From a practical perspective, our findings of significantly better absolute and relative YBT-LQ/UQ values in trained compared to untrained youth and the good discriminative validity indicate that both tests can be used to distinguish between young athletes and age -/sex-matched controls based on dynamic balance (YBT-LQ) and shoulder mobility/stability (YBT-UQ) data. Consequently, both testing procedures can be used to discriminate persons with higher compared to lower levels of performance. This allows for the possibility to offer specifically tailored sport programs to support growing-ups according to their individual performance level, e.g., fitness promoting programs for low fit individuals and young athlete training regimens for high fit subjects.

Of note, our findings are limited to two cohorts (i.e., soccer players and swimmers). Both groups represent cohorts that are used to control their postural stability in challenging situations (i.e., soccer players) or to maintain mobility/stability in their pectoral girdle and upper extremities (i.e., swimmers). Consequently, our findings cannot be generalized to other populations or sports and further research is needed to examine athletes from different disciplines or other cohorts.

\section{Conclusions}

We investigated the discriminative validity of absolute and relative YBT-LQ/UQ performance between healthy trained (i.e., soccer players and swimmers) and untrained youth (i.e., age-/sex-matched controls) and found significantly better values for the former one as well as good discriminative validity for both tests. Our findings indicate that both, the YBT-LQ and the YBT-UQ are suitable field tests to effectively differentiate between trained and age-/sex-matched untrained youth based on dynamic balance (YBT-LQ) and shoulder mobility/stability (YBT-UQ) data.

\section{Abbreviations \\ AL: Arm length; ANOVA: Analysis of variance; AT: Anterior; AUC: Area under the receiver operator characteristic curve; BMI: Body mass index; \\ CS: Composite score; IL: Inferolateral; LL: Leg length; MD: Medial; PHV: Peak height velocity; PL: Posterolateral; PM: Posteromedial; ROC: Receiver operator characteristic curve; SL: Superolateral; YBT-LQ: Lower Quarter Y Balance Test; YBT-UQ: Upper Quarter Y Balance Test}

\section{Acknowledgements}

Not applicable.

\section{Authors' contributions}

GS, RB, and TM designed the research question. GS planned and conducted the testings and data collections. GS and TM analyzed the data. GS, RB, and TM wrote the main parts of the manuscript. All authors contributed to critical review of draft manuscripts and approved the final manuscript.

\section{Funding}

We acknowledge support by the Open Access Publication Fund of the University of Duisburg-Essen. The funding body is independent of the design of the study and collection, analysis, and interpretation of data and in writing the manuscript. Open Access funding enabled and organized by Projekt DEAL.

\section{Availability of data and materials}

The data generated and analyzed during the present study are not publicly available due to ethical restrictions but are available from the corresponding author upon reasonable request.

\section{Ethics approval and consent to participate}

Ethical permission was given by the Human Ethics Committee at the University of Duisburg-Essen, Faculty of Educational Sciences. Participants assent and parents' written informed consent were obtained prior to the start of the study.

\section{Consent for publication}

Not applicable.

\section{Competing interests}

The authors declare that they have no competing interests. TM is an Associate Editor of the BMC Sports Science, Medicine, and Rehabilitation journal.

\section{Author details}

${ }^{1}$ Division of Movement and Training Sciences/Biomechanics of Sport, University of Duisburg-Essen, Gladbecker Str. 182, 45141 Essen, Germany. 
${ }^{2}$ Department of Health and Social Affairs, FHM Bielefeld - University of Applied Sciences, Bielefeld, Germany.

Received: 10 August 2020 Accepted: 9 November 2020

Published online: 03 December 2020

\section{References}

1. Read PJ, Oliver JL, De Ste Croix MBA, Myer GD, Lloyd RS. A review of fieldbased assessments of neuromuscular control and their utility in male youth soccer players. J Strength Cond Res. 2019;33:283-99.

2. Borms D, Cools A. Upper-extremity functional performance tests: reference values for overhead athletes. Int J Sports Med. 2018;39:433-41.

3. Butler RJ, Southers C, Gorman PP, Kiesel KB, Plisky PJ. Differences in soccer players' dynamic balance across levels of competition. J Athl Train. 2012;47: 616-20.

4. Westrick RB, Miller JM, Carow SD, Gerber JP. Exploration of the y-balance test for assessment of upper quarter closed kinetic chain performance. Int J Sports Phys Ther. 2012;7:139-47.

5. Schwiertz G, Brueckner D, Beurskens R, Muehlbauer T. Lower quarter $Y$ balance test performance: reference values for healthy youth aged 10 to 17 years. Gait Posture. 2020;80:148-54.

6. Teyhen DS, Riebel MA, McArthur DR, Savini M, Jones MJ, Goffar SL, Kiesel KB, Plisky PJ. Normative data and the influence of age and gender on power, balance, flexibility, and functional movement in healthy service members. Mil Med. 2014;179:413-20.

7. Butler R, Arms J, Reiman M, Plisky P, Kiesel K, Taylor D, Queen R. Sex differences in dynamic closed kinetic chain upper quarter function in collegiate swimmers. J Athl Train. 2014;49:442-6.

8. Fusco A, Giancotti GF, Fuchs PX, Wagner H, da Silva RA, Cortis C. Y balance test: are we doing it right? J Sci Med Sport. 2020:23:194-9.

9. Gribble PA, Hertel J. Considerations for normalizing measures of the star excursion balance test. Measure Phys Education Exerc Sci. 2003;7:89-100

10. Wilson BR, Robertson KE, Burnham JM, Yonz MC, Ireland ML, Noehren B. The relationship between hip strength and the $Y$ balance test. J Sport Rehabil. 2018;27:445-50.

11. Read PJ, Oliver JL, Myer GD, Farooq A, De Ste CM, Lloyd RS. Utility of the anterior reach $Y$-balance test as an injury risk screening tool in elite male youth soccer players. Phys Ther Sport. 2020;45:103-10.

12. Kim $Y$, Lee JM, Wellsandt $E$, Rosen AB. Comparison of shoulder range of motion, strength, and upper quarter dynamic balance between NCAA division I overhead athletes with and without a history of shoulder injury. Phys Ther Sport. 2020;42:53-60

13. Bullock GS, Arnold TW, Plisky PJ, Butler RJ. Basketball players' dynamic performance across competition levels. J Strength Cond Res. 2018;32:352833.

14. Bullock GS, Brookreson N, Knab AM, Butler RJ. Examining fundamental movement competency and closed-chain upper-extremity dynamic balance in swimmers. J Strength Cond Res. 2017:31:1544-51.

15. Krysak S, Harnish CR, Plisky PJ, Knab AM, Bullock GS. Fundamental movement and dynamic balance disparities among varying skill levels in golfers. Int J Sports Phys Ther. 2019;14:537-45

16. Balyi I, Way R, Higgs C. Long-term athlete development. Champaign: Human Kinetics; 2013.

17. Moore SA, McKay HA, Macdonald H, Nettlefold L, Baxter-Jones AD, Cameron $\mathrm{N}$, Brasher PM. Enhancing a somatic maturity prediction model. Med Sci Sports Exerc. 2015;47:1755-64.

18. Plisky PJ. Y balance test home study course; 2010.

19. Plisky PJ, Gorman PP, Butler RJ, Kiesel KB, Underwood FB, Elkins B. The reliability of an instrumented device for measuring components of the star excursion balance test. N Am J Sports Phys Ther. 2009;4:92-9.

20. Schwiertz G, Brueckner D, Schedler S, Kiss R, Muehlbauer T. Performance and reliability of the lower quarter $Y$ balance test in healthy adolescents from grade 6 to 11. Gait Posture. 2018;67:142-6.

21. Schwiertz G, Brueckner D, Schedler S, Kiss R, Muehlbauer T. Reliability and minimal detectable change of the upper quarter $Y$ balance test in healthy adolescents aged 12 to 17 years. Int J Sports Phys Ther. 2019:14:927-34

22. Cohen J. Statistical power for the behavioral sciences. Hillsdale: Erlbaum; 1988

23. Deyo RA, Centor RM. Assessing the responsiveness of functional scales to clinical change: an analogy to diagnostic test performance. J Chronic Dis. 1986:39:897-906.
24. Butler RJ, Bullock G, Arnold T, Plisky P, Queen R. Competition-level differences on the lower quarter Y-balance test in baseball players. J Athl Train. 2016;51:997-1002.

25. McCann RS, Kosik KB, Beard MQ, Terada M, Pietrosimone BG, Gribble PA Variations in star excursion balance test performance between high school and collegiate football players. J Strength Cond Res. 2015;29:2765-70.

26. Engquist KD, Smith CA, Chimera NJ, Warren M. Performance comparison of student-athletes and general college students on the functional movement screen and the $Y$ balance test. J Strength Cond Res. 2015;29:2296-303.

27. Araujo CG, Scharhag J. Athlete: a working definition for medical and health sciences research. Scand J Med Sci Sports. 2016:26:4-7.

28. Murtagh CF, Brownlee TE, Rienzi E, Roquero S, Moreno S, Huertas G, Lugioratto G, Baumert P, Turner DC, Lee D, et al. The genetic profile of elite youth soccer players and its association with power and speed depends on maturity status. PLoS One. 2020;15:e0234458.

29. Stastny $P$, Lehnert M, De Ste CM, Petr M, Svoboda Z, Maixnerova E, Varekova R, Botek M, Petrek M, Kocourkova L, et al. Effect of COL5A1, GDF5, and PPARA genes on a movement screen and neuromuscular performance in adolescent team sport athletes. J Strength Cond Res. 2019;33:2057-65.

30. Nakata H, Yoshie M, Miura A, Kudo K. Characteristics of the athletes' brain: evidence from neurophysiology and neuroimaging. Brain Res Rev. 2010;62: 197-211.

31. Yarrow K, Brown P, Krakauer JW. Inside the brain of an elite athlete: the neural processes that support high achievement in sports. Nat Rev Neurosci. 2009;10:585-96.

\section{Publisher's Note}

Springer Nature remains neutral with regard to jurisdictional claims in published maps and institutional affiliations.
Ready to submit your research? Choose BMC and benefit from:

- fast, convenient online submission

- thorough peer review by experienced researchers in your field

- rapid publication on acceptance

- support for research data, including large and complex data types

- gold Open Access which fosters wider collaboration and increased citations

- maximum visibility for your research: over $100 \mathrm{M}$ website views per year

At BMC, research is always in progress.

Learn more biomedcentral.com/submissions 